\title{
Optical absorption and magnetic circular dichroism spectra of thiouracils: a quantum mechanical study in solution
}

Martínez-Fernández , L.; Fahleson, Tobias; Norman, Patrick; Santoro, Fabrizio; Coriani, Sonia; Improta, Roberto

Published in:

Photochemical \& Photobiological Sciences

Link to article, DOI:

$10.1039 / \mathrm{c} 7 \mathrm{pp} 00105 \mathrm{c}$

Publication date:

2017

Document Version

Peer reviewed version

Link back to DTU Orbit

Citation (APA):

Martínez-Fernández , L., Fahleson, T., Norman, P., Santoro, F., Coriani, S., \& Improta, R. (2017). Optical absorption and magnetic circular dichroism spectra of thiouracils: a quantum mechanical study in solution. Photochemical \& Photobiological Sciences, 16(9), 1415-1423. https://doi.org/10.1039/c7pp00105c

\section{General rights}

Copyright and moral rights for the publications made accessible in the public portal are retained by the authors and/or other copyright owners and it is a condition of accessing publications that users recognise and abide by the legal requirements associated with these rights.

- Users may download and print one copy of any publication from the public portal for the purpose of private study or research.

- You may not further distribute the material or use it for any profit-making activity or commercial gain

- You may freely distribute the URL identifying the publication in the public portal 


\title{
Journal Name
}

\section{ARTICLE}

\section{Optical Absorption and Magnetic Circular Dichroism spectra of thiouracils: a quantum mechanical study in solution}

Received 00th January 20xx, Accepted 00th January 20xx

\begin{abstract}
L. Martinez-Fernandez, ${ }^{{ }^{a}}$ T. Fahleson, ${ }^{b}$ P. Norman, ${ }^{b}$ F. Santoro, ${ }^{c}$ S. Coriani $*^{d}$ and R. Improta ${ }^{a, e}$
The excited electronic states of 2-thiouracil, 4-thiouracil and 2,4-dithiouracil, the analogues of uracil where the carbonyl oxygens are substituted by sulphur atoms, have been investigated by computing magnetic circular dichroism (MCD) and one-photon absorption (OPA) at the Time-Dependent Density Functional Theory level. Special attention has been paid to solvent effects, included by a mixed discrete/continuum model, and to analyse how our results depend on the adopted TD functional (CAM-B3LYP and B3LYP). Whereas including solvent effect does not dramatically impact the MCD and OPA spectra, though improving the agreement with the experimental spectra, the performances of CAM-B3LYP and B3LYP are remarkably different. CAM-B3LYP well captures the effect of thionation on the uracil excited states and provides spectra in good agreement with the experiments, whereas B3LYP shows some deficiency in describing 2-TU and 2,4-DTU spectra, though being more accurate than CAM-B3LYP for 4-TU.
\end{abstract}

\section{Introduction}

Even though the use of light with therapeutic purposes is ancient, ${ }^{1}$ modern photodynamic therapy of cancer (PDT) was initiated in $1960^{\prime 2} s^{2,3}$ and it wasn't until $1975^{4}$ that the first human trial was performed..$^{5-7}$ PDT is a two-step treatment that combines light and a photosensitizer (PS) to cause cell death. First, the patient is administrated with the PS and after some incubation period, the PS is activated by exposure to a certain wavelength of light. Triplet excited states of the PSs transfer energy to molecular oxygen, generating singlet oxygen and other reactive oxygen species (ROS) that mediate cellular toxicity. Despite being a significant medical advance in the last century, ${ }^{5}$ several aspects of PDT can be hugely improved, and one of the key points is the development of more effective PSs. Porphyrins, the most used PSs at the moment, poorly absorb light and suffer from important side effects. ${ }^{8,9}$

Thio-substituted DNA nucleobases, the thiobases, recently emerged as very promising candidates, and are actively tested against different kinds of cancer. ${ }^{10-18}$ Their strong UVA absorption plus very efficient triplet state population are the basis for their use as PS. ${ }^{19-44}$ Besides these unique photosensitizing properties, thiobases present further applications in biomedical fields. In particular, 4-thiouracil, has been used as site-specific photocrosslinking agent ${ }^{45-49}$ to tag either RNA molecules or proteins. In this important structuralbiological function, 4-thiouracil forms pyrimidine-pyrimidone products between the $\mathrm{C}=\mathrm{S}$ and $\mathrm{C} 5=\mathrm{C} 6$ bonds. ${ }^{50,51}$ On the other

\footnotetext{
${ }^{a .}$ Istituto di Biostrutture e Bioimmagini-CNR, Via Mezzocannone 6, I-80134 Napoli, Italy

b. KTH Royal Institute of Technology, SE-10044 Stockholm, Sweden

Istituto di Chimica dei Composti Organometallici (ICCOM-CNR), Area della

Ricerca del CNR, Via Moruzzi 1, I-56124 Pisa, Italy

${ }^{d .}$ Department of Chemistry, Technical University of Denmark, DK-2800 Kgs. Lyngby, Denmark

e. LIDYL, CEA, CNRS, Université Paris Saclay, F-91191 Gif-sur-Yvette, France
}

hand, 2-thiouracil is instead widely used as therapeutic agent in thyroid pathologies ${ }^{52}$ and its ability to complex with metal ions ${ }^{53}$ has been exploited as antidote for mercury poisoning. ${ }^{54}$

Most of these biomedical applications of thiobases exploit their unique photophysical properties. Oxygen-by-sulphur substitutions red-shift the absorption maximum and enhance intersystem crossing rates compared to canonical bases. ${ }^{23,24,}$ $32-34,37,38,55$ Interestingly, these changes depend on the substitution position (for instance, the effects are larger for 4thiopyrimidines) whereas double thionation (i.e. in position 2 and 4) does not have a dramatic effect. ${ }^{33,34}$ The therapeutic interest of thiobases has also motivated many theoretical studies of their excited state properties. ${ }^{19,} 20,25-28,31,40,42,44,56$ These works have explained that it is the presence of the $\mathrm{C}=\mathrm{S}$ moiety that leads to the red-shift of the absorption spectrum and to the enhancement of the spin orbit couplings. These studies also showed that dark singlet minima reached after internal conversion (IC) from the spectroscopic state, are essential for their use as PS, since they act as doorway for triplet population. $^{20,28,29,31}$ Despite their importance, the energy of the dark states at the Franck-Condon (FC) region and how it is affected by solvent is often overlooked, in part due to their non detectability in absorption spectroscopy. However, the energy gap between dark and excited states at the FC region can be essential since, as explained above, the IC process is the first step towards triplet population. Similarly, for what concerns canonical nucleobases, despite many recent computational efforts, in several cases the relative stability of dark and bright excited states is still elusive, leading to strong disagreement on the involvement of the former in the photoactivated dynamics. ${ }^{57-60}$ Actually, in some cases, as for adenine, the debate even involves the energy ordering of bright excited states. ${ }^{61,62}$ Magnetic circular dichroism (MCD), thanks to the different excitation mechanism and its signed 
nature can be helpful to shed light in these situations, as in our previous study on adenine. ${ }^{61}$

In the present contribution we report a detailed study of the electronic excited states of thiouracils, paying attention to how different thionation positions and solvation models affect their optical and MCD spectra. We have computed the optical and MCD spectra of 2-thiouracil (2-TU), 4-thiouracil (4-TU) and 2,4dithiouracil (2,4-DTU) in vacuo and in water using the polarizable continuum model (PCM). Furthermore, explicit water molecules were also considered, since previous studies indicated that their inclusion is fundamental to reproduce the relative energy of bright and dark excited states in pyrimidines. $^{63}$ Our analysis is based on the results of TD-DFT calculations, obtained exploiting two commonly adopted density functionals, namely CAM-B3LYP and B3LYP.

\section{Computational Details}

Geometry Optimization. The ground state of 2-TU, 4-TU and 2,4DTU have been optimized at the $B 3 \mathrm{LYP}^{64} / \mathrm{cc}^{-p V T Z} \mathrm{p}^{65}$ level of theory. All geometry optimizations were performed with Gaussian09. ${ }^{66}$

Solvent. Solvent effects have been modelled using three different approaches: (1) implicitly, by using the Integral Equation Formulation of the Polarizable Continuum Model (IEF-PCM ) ${ }^{67-75}$; (2) explicitly, including $4 \bullet \mathrm{H}_{2} \mathrm{O}$ molecules into the calculations, and (3) resorting to a mixed implicit/explicit scheme where both PCM and $4 \bullet \mathrm{H}_{2} \mathrm{O}$ are considered. Non-equilibrium solvation was adopted in the IEF-PCM calculations. The number of water molecules (four) defining the first solvation shell was selected based on experimental evidences ${ }^{76,77}$ and on the success of this model in previous computational studies of uracil photophysics. ${ }^{78-81}$ The role of solvent dynamics was not considered throughout this study.

Figure 1. Schematic drawing and atom labelling of Uracil and its corresponding thio-derivatives, 2-thiouracil (2-TU), 4-thiouracil (4-TU) and 2,4-dithiouracil (2,4-DTU). See 2-TU as example of the adopted computational model for the systems $4 \bullet \mathrm{H}_{2} \mathrm{O}$.
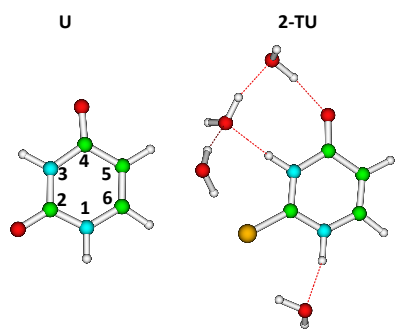

4-TU

2,4-DTU
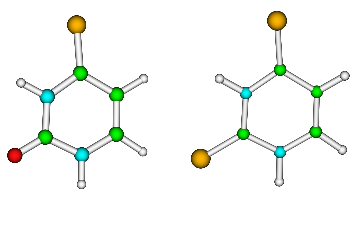

One-Photon Absorption and Magnetic Circular Dichroism Spectra. The one-photon absorption (OPA) and MCD spectra were computed using both B3LYP and CAM-B3LYP ${ }^{82}$ functionals, adopting the augcc-pVDZ ${ }^{83}$ basis set. The spectra were obtained directly using the complex-polarization-propagator (CPP) approach, ${ }^{84-86}$ as implemented within the Dalton ${ }^{87}$ program package. By explicitly accounting for the finite lifetime of the excited states, CPP allows to solve possible issues related to the divergence of standard quadratic response calculations in cases of quasi degeneracies. In the theory, the empirical parameter $v$ represents the inverse lifetime of vibronic states, and it results in a Lorentzian spectral broadening. In implementations of the theory that only accounts for electronic responses in the system (such as ours), one typically adopts numerical values of $\gamma$ that exceed inverse singlet state lifetimes (a value of $\sim 0.1 \mathrm{eV}$ is often used) since, in this manner, one can mimic the (vibrational and solvent) spectral broadening seen in the experiment. Here, as done in precedent works, we chose the same phenomenological parameter for all states and assigned it a value of $1000 \mathrm{~cm}^{-1}$. This value has earlier been proven a reasonable choice that yields smooth spectra (and therefore robust results) and intensities comparable to experiment (too small $\gamma$ would give rise to unphysically narrow and intense peaks). On the other hands this choice avoids linewidths so large to prevent the identification of the different peaks corresponding to different states. The same computational strategy and broadening parameter were already adopted in our previous studies on a number of purine and pyrimidine nucleobases predicting OPA and MCD spectra in general agreement with experiment. $^{61,80}$ It rests assured that the experimental width of the spectra is primarily due to vibrational and solvent broadening mechanisms, which could be theoretically accounted for [see, e.g., refs ${ }^{88-91}$ ] in more refined ways than done here, but this goes beyond the scope of the present work.

Computed OPA and MCD spectra were reported as decadic molar extinction coefficient, $\varepsilon$, and as extinction coefficient anisotropy $\Delta \varepsilon$, respectively, in the standard units $\left[\mathrm{M}^{-1} \mathrm{~cm}^{-1}\right]$ and $\left[\mathrm{M}^{-1} \mathrm{~cm}^{-1} \mathrm{~T}^{-1}\right]$. Experimental OPA and MCD spectra were taken from ref. ${ }^{92}$, where OPA was reported as molar extinction coefficient $\varepsilon$ in standard units, whereas MCD was given as molar ellipticity $[\vartheta]_{M}$, formally in units of deg $\mathrm{M}^{-1} \mathrm{~m}^{-1} \mathrm{~T}^{-1}$. To convert the experimental MCD data in ref ${ }^{92}$ into $\Delta \varepsilon$ in standard units $\mathrm{M}^{-1} \mathrm{~cm}^{-1} \mathrm{~T}^{-1}$, they should be divided by $3.29810^{5}$, yielding intensities about six orders of magnitudes smaller than the computed ones. As thoroughly discussed in the SI, we have robust elements to believe that the MCD experimental units in ref ${ }^{92}$ were misprinted and that the scaling factor missing is exactly $10^{6}$. Adopting a prudential approach, in this study we prefer however to focus our comparison the signs, relative intensity and energy separations of the different peaks. We therefore report the experimental spectra normalized so to have the most intense peak (absolute values) equal to 1 . A comparison of the MCD intensities is instead given in the SI.

All the calculations have been performed on the thio-keto tautomers of thiouracils.

\section{Results}

2-Thiouracil (2-TU). Below $6 \mathrm{eV}, 2$-TU presents two bright and three dark excited states (Table 1). At the CAM-B3LYP level (in gas phase, GP) the bright $\pi_{c c} \pi^{*}$ cs and $\pi_{c c} \pi^{*}$ cc excited states are separated by $\sim 0.4 \mathrm{eV}$ and have similar oscillator strengths. Both excitations involve $\pi$ and $\pi *$ orbitals that are delocalized over the 2-TU aromatic ring, but exhibit important contributions also from the $\mathrm{C}=\mathrm{S}$ bond. In particular, the LUMO is reminiscent of the corresponding orbital of uracil, with strong antibonding character with respect to the $\mathrm{C} 5$ and $\mathrm{C} 6$ double bond, but it also exhibits a strong antibonding contribution between $\mathrm{C} 2$ and $\mathrm{S}$ bond. The three dark states correspond to different combinations of the possible excitations from both lone pairs ( $\mathrm{O}$ and $\mathrm{S}$ ) to the two lowest energy $\pi *$ orbitals. $S_{1}$ corresponds to a dark excited state, with main contribution from the $\mathrm{S}$ lone pair. 
Inclusion of solvent effects has a very small impact on the nature and the relative energy of the bright excited states, but significantly destabilizes the dark excited states (by 0.3 0.4 eV), in analogy with what happens for natural pyrimidines. ${ }^{57,93,94}$ As a consequence, $\mathrm{S}_{1}$, which in the gas phase is $\sim 0.8 \mathrm{eV}$ more stable that the lowest energy bright state, gets much closer in energy to the latter ( 0.4). The computed absorption spectrum is in good agreement with the experimental one (Figure $2 \mathrm{~d}$ ), ${ }^{33}, 92$ which shows a very broad band with maximum at $\sim 265 \mathrm{~nm}(4.68 \mathrm{eV})$ and a feature at $\sim 290 \mathrm{~nm}(4.27$ $\mathrm{eV})$, which we assign to $\pi_{\mathrm{cc}} \pi^{*} \mathrm{cc}$ and $\pi_{\mathrm{cc}} \pi^{*}$ cs, except for a uniform blue-shift of $<0.4 \mathrm{eV}$. As discussed in several previous contributions, this blue-shift may be partially due to the lack of vibrational contribution in the computed spectra. ${ }^{63,95}$

The experimental MCD spectrum of 2-TU presents two bands of similar intensity but opposite sign: Band I peaked at $4.27 \mathrm{eV}$ $(290 \mathrm{~nm})$ and Band II peaked at $4.67 \mathrm{eV}$ (265 nm). Their maxima are separated by $\sim 0.4 \mathrm{eV}$ (Figure 2a). Our computed MCD spectra nicely match the shape of the experimental spectra, but with the overall blue-shift commented above in the absorption spectra. We can thus assign the two experimental peaks to $\pi_{\mathrm{CC}} \pi^{*}$ cS and $\pi_{\mathrm{CC}} \pi^{*}{ }_{\mathrm{CC}}$ states, respectively. As expected, the band positions of the different computed MCD spectra are not dramatically affected by the inclusion of solvent effect, which, however, improves the agreement with the relative intensities of the experimental peaks. While in the gas phase, Band II is smaller than Band I, in water the two peaks have similar intensity, as in the experiment (Figure 2a).

Table 1. 2-TU Vertical absorption energies $(\Delta E$, in $e V)$ and oscillator strengths (f) for the different solvation models, calculated at the TD-CAMB3LYP/aug-cc-pVDZ and TD-B3LYP/aug-cc-pVDZ level of theory.

\begin{tabular}{|c|c|c|c|c|c|c|c|c|}
\hline 2-TU & \multicolumn{2}{|c|}{ GP } & \multicolumn{2}{|c|}{ PCM } & \multicolumn{2}{|c|}{$4 \cdot \mathrm{H}_{2} \mathrm{O}$} & \multicolumn{2}{|c|}{$\mathrm{PCM}+4 \cdot \mathrm{H}_{2} \mathrm{O}$} \\
\hline $\begin{array}{l}\text { CAM- } \\
\text { B3LYP }\end{array}$ & $\Delta \mathrm{E}$ & $f$ & $\Delta \mathrm{E}$ & $f$ & $\Delta \mathrm{E}$ & $f$ & $\Delta \mathrm{E}$ & $f$ \\
\hline $\begin{array}{r}\mathrm{S}_{1} \mathrm{LP}_{\mathrm{s}} \pi^{*}{ }^{*} \mathrm{cs} \\
\mathrm{LP} \mathrm{P}_{\mathrm{s}} \pi^{*} \mathrm{cc}\end{array}$ & 3.86 & 0.00 & 4.09 & 0.00 & 4.03 & 0.00 & 4.22 & 0.00 \\
\hline $\mathrm{S}_{2} \pi_{\mathrm{cc}} \pi^{*} \mathrm{cs}$ & 4.62 & 0.20 & 4.64 & 0.37 & 4.54 & 0.13 & 4.64 & 0.27 \\
\hline $\mathrm{S}_{3} \pi_{\mathrm{cc}} \pi^{*} \mathrm{cc}$ & 5.08 & 0.17 & 5.08 & 0.11 & 4.99 & 0.19 & 5.06 & 0.14 \\
\hline $\begin{array}{r}\mathrm{S}_{4} \mathrm{LP}_{\mathrm{s}} \pi^{*} \mathrm{cc} \\
\mathrm{LP} \mathrm{P}_{\mathrm{s}} \pi^{*}{ }^{* \mathrm{cs}} \\
\mathrm{LP} \mathrm{P}_{\mathrm{o}}{ }^{*} \mathrm{cs} \\
\mathrm{LP} \mathrm{P}_{\mathrm{o}} \pi^{*}{ }^{\mathrm{c}}{ }^{2}\end{array}$ & 4.92 & 0.00 & 5.20 & 0.00 & 5.07 & 0.00 & 5.33 & 0.00 \\
\hline $\begin{array}{c}S_{5} L P_{s} \pi^{*} c c \\
L P_{s} \pi^{*}{ }^{*} \\
L P_{o} \pi^{*}{ }^{*} c s \\
L P_{0} \pi^{*}{ }^{c c}\end{array}$ & 5.43 & 0.00 & 5.69 & 0.00 & 5.45 & 0.00 & 5.72 & 0.00 \\
\hline B3LYP & $\Delta \mathrm{E}$ & $f$ & $\Delta \mathrm{E}$ & $f$ & $\Delta \mathrm{E}$ & $f$ & $\Delta \mathrm{E}$ & $f$ \\
\hline $\begin{array}{r}\mathrm{S}_{1} \mathrm{LP}_{\mathrm{s}} \pi^{*} \mathrm{cs} \\
\mathrm{LP} \mathrm{P}_{\mathrm{s}} \pi^{*} \mathrm{cc}\end{array}$ & 3.57 & 0.00 & 3.78 & 0.00 & 3.65 & 0.00 & 3.88 & 0.00 \\
\hline $\mathrm{S}_{2} \pi_{\mathrm{cc}} \pi^{*} \mathrm{cs}$ & 4.07 & 0.05 & 4.20 & 0.11 & 3.96 & 0.03 & 4.20 & 0.08 \\
\hline $\begin{array}{r}\mathrm{S}_{3} \mathrm{LP}_{\mathrm{s}} \pi^{*} \mathrm{cc} \\
\mathrm{LP} \mathrm{P}_{\mathrm{s}} \pi^{*} \mathrm{cs}\end{array}$ & 4.14 & 0.00 & 4.35 & 0.00 & 4.17 & 0.00 & 4.41 & 0.00 \\
\hline $\begin{array}{r}\mathrm{S}_{4} \mathrm{LP}_{\mathrm{o}} \pi^{*} \mathrm{cs} \\
\mathrm{LP} \mathrm{P}_{\mathrm{o}} \pi^{*} \mathrm{cc}\end{array}$ & 4.77 & 0.00 & 5.05 & 0.00 & 4.64 & 0.00 & 5.15 & 0.00 \\
\hline$S_{5} \pi_{c c} \pi^{*}{ }_{c c}$ & 4.85 & 0.27 & 4.78 & 0.33 & 4.76 & 0.27 & 4.74 & 0.32 \\
\hline & & & & & & & & \\
\hline
\end{tabular}

The B3LYP functional provides a different picture than the one, described above, provided by CAM-B3LYP. The main effect is the destabilization of the $\pi_{C C} \pi^{*}{ }_{c C}$ state, which does not correspond to $S_{3}$ but to $S_{5}$, increasing the energy gap with respect to $S_{2}$. Furthermore, the relative intensity of the two lowest bright states is reversed compared to CAM-B3LYP, B3LYP predicting that the $\pi_{C C} \pi^{*}{ }_{C C}$ is much more intense than $S_{2}$, especially when PCM is not included. The agreement with the experimental absorption spectra worsens (Figure 3d) since B3LYP predicts two well-separated bands with different intensities, instead of a single broad peak. On the other hand, the adoption of a slightly larger value for the phenomenological broadening would have led to a computed spectrum in qualitative agreement with the experiments, potentially obscuring the possible inaccuracies of B3LYP, which, are instead apparent when computing the MCD spectrum.

The B3LYP computed MCD spectrum does not indeed agree with the experimental one (Figure 3a), except for the presence of a negative feature on the red-wing. When PCM is not included a $n$ Rydberg transition falls close in energy to the second bright-excited state, leading to the appearance of negative/positive double feature. This feature is displaced at higher energy by PCM. However, in this case, a vanishingly small negative feature is associated to the very intense $\pi_{\mathrm{CC}} \pi^{*}{ }_{\mathrm{CC}}$ transition.

4-Thiouracil (4-TU). The impact of thio-substitution in position 4 on the frontier orbitals of uracil is very different from the one of 2thionation, since sulphur participates to a 'butadiene' like conjugation with $\mathrm{C} 5=\mathrm{C} 6$ double bond. As a consequence, sulphur strongly contributes to both the $\pi$ HOMO and the $\pi^{*}$ LUMO, which are involved in lowest energy bright transition $\left(\pi_{c C} \pi^{*}{ }_{C S}\right)$. This transition (in GP) is significantly more intense (one order of magnitude) than, and red-shifted $(4.34 \mathrm{eV})$ with respect to, the $\pi_{C S} \pi^{*}{ }_{C S}(5.22 \mathrm{eV})$ and $\pi_{C C} \pi^{*}{ }_{C C}(5.59 \mathrm{eV})$ transitions. Compared with the bright states appearing in 2-TU, a new bright state $\left(\pi_{\mathrm{CS}} \pi^{*}{ }_{\mathrm{CS}}\right)$ appears characterized by a participation of the $\mathrm{C}=\mathrm{S}$ bond to both bonding and antibonding orbitals.

Concerning the dark states, the energy gap between $\mathrm{S}_{1}\left(\mathrm{LP}_{\mathrm{S}} \pi^{*}{ }_{\mathrm{CS}}\right)$ and $\mathrm{S}_{2}\left(\pi_{\mathrm{Cc}} \pi^{*}{ }_{\mathrm{CS}}\right)$ in GP is significantly larger than in 2-TU, whereas the separation between the second dark state $S_{5}\left(\operatorname{LP}_{5} \pi^{*}{ }^{*} C\right)$ and the closest bright state $\left(\mathrm{S}_{4}\right)$ is maintained. Another difference with 2-TU concerns $L P_{0}$, which does not participate in any of the low-lying excited states of 4-TU.

Water strongly destabilizes the $S_{1}\left(L P_{s} \pi^{*} c s\right)$ dark state, while increasing the stability $(0.2 \mathrm{eV})$ and OPA intensity of $S_{2}\left(\pi_{\mathrm{CC}} \pi^{*}{ }_{\mathrm{CS}}\right)$ (especially when PCM is considered). As a consequence, as it happens for 2-TU, these two states are much closer in water than in the gas phase. Solvent effect on $\mathrm{S}_{3}$ and $\mathrm{S}_{4}$ is instead much smaller.

The computed OPA CAM-B3LYP spectrum in water are in good agreement with the experimental one (Figure 2e), ${ }^{33,92}$ the latter exhibiting a very intense peak at $\sim 330 \mathrm{~nm}(3.75 \mathrm{eV})$ which we assign to the $\pi_{c c} \pi^{*}$ cs state, and a broad and weak band at 230-270 nm (maximum at $\sim 240 \mathrm{~nm}$, i.e. $5.16 \mathrm{eV}$ ), which we assign to $\pi_{c S} \pi^{*}$ cs and $\pi_{c c} \pi^{*}{ }_{c c}$, though our calculations underestimate the relative intensity of this peak. Also in this case the computed vertical 
absorption energies (VAE) are blue-shifted by $0.4-0.5 \mathrm{eV}$ with respect to the experimental maxima.

Table 2. 4-TU Vertical absorption energies $(\Delta \mathrm{E}$, in $\mathrm{eV})$ and oscillator strengths (f) for the different solvation models calculated at the TD-CAM-B3LYP/aug-CCpVDZ and TD-B3LYP/aug-cc-pVDZ levels of theory.

\begin{tabular}{|c|c|c|c|c|c|c|c|c|}
\hline 4-TU & \multicolumn{2}{|c|}{ GP } & \multicolumn{2}{|c|}{ PCM } & \multicolumn{2}{|c|}{$4 \cdot \mathrm{H}_{2} \mathrm{O}$} & \multicolumn{2}{|c|}{$\mathrm{PCM}+4 \cdot \mathrm{H}_{2} \mathrm{O}$} \\
\hline $\begin{array}{l}\text { CAM- } \\
\text { B3LYP }\end{array}$ & $\Delta \mathrm{E}$ & $f$ & $\overline{\Delta E}$ & $\bar{f}$ & $\overline{\Delta E}$ & $f$ & $\Delta \mathrm{E}$ & $\bar{f}$ \\
\hline $\begin{array}{r}\mathrm{S}_{1} \mathrm{LP}_{\mathrm{s}} \pi^{*} \mathrm{cs} \\
\mathrm{LP} \mathrm{P}_{\mathrm{s}} \pi^{*} \mathrm{cc}\end{array}$ & 3.03 & 0.00 & 3.37 & 0.00 & 3.28 & 0.00 & 3.54 & 0.00 \\
\hline $\mathrm{S}_{2} \pi_{c c} \pi^{*} \mathrm{cs}$ & 4.34 & 0.34 & 4.21 & 0.42 & 4.24 & 0.38 & 4.20 & 0.47 \\
\hline $\mathrm{S}_{3} \pi_{\mathrm{cs}} \boldsymbol{\pi}^{*} \mathrm{cs}$ & 5.22 & 0.01 & 5.22 & 0.02 & 5.19 & 0.01 & 5.24 & 0.02 \\
\hline $\mathrm{S}_{4} \pi_{c c} \pi^{*} c c$ & 5.59 & 0.04 & 5.67 & 0.06 & 5.48 & 0.04 & 5.61 & 0.04 \\
\hline $\begin{array}{r}\mathrm{S}_{5} \mathrm{LP}_{\mathrm{s}} \pi^{*} \mathrm{cc} \\
L P_{\mathrm{s}} \pi^{*}{ }_{\mathrm{cs}}\end{array}$ & 5.54 & 0.00 & 5.83 & 0.00 & 5.64 & 0.00 & 5.90 & 0.00 \\
\hline $\mathrm{S}_{6} \pi_{\mathrm{cc}} \mathrm{Rydb}$ & 5.57 & 0.00 & 5.90 & 0.01 & 5.81 & 0.00 & 5.96 & 0.02 \\
\hline $\mathrm{S}_{7} \mathrm{LP}_{\mathrm{s}} \mathrm{Rydb}$ & 5.70 & 0.03 & 5.88 & 0.10 & 5.96 & 0.05 & 6.04 & 0.09 \\
\hline B3LYP & $\overline{\Delta E}$ & $\bar{f}$ & $\overline{\Delta \mathrm{E}}$ & $\bar{f}$ & $\overline{\Delta E}$ & $\bar{f}$ & $\overline{\Delta \mathrm{E}}$ & $\bar{f}$ \\
\hline $\begin{array}{l}S_{1} L_{\mathrm{S}_{\mathrm{s}}} \pi^{*} \mathrm{cs} \\
\mathrm{S}_{2} \pi_{\mathrm{cc}} \pi^{*} \mathrm{cs}\end{array}$ & $\begin{array}{l}2.79 \\
4.17\end{array}$ & $\begin{array}{l}0.00 \\
0.24\end{array}$ & $\begin{array}{l}3.08 \\
4.09\end{array}$ & $\begin{array}{l}0.00 \\
0.36\end{array}$ & $\begin{array}{l}2.99 \\
4.08\end{array}$ & $\begin{array}{l}0.00 \\
0.28\end{array}$ & $\begin{array}{l}3.24 \\
4.08\end{array}$ & $\begin{array}{l}0.00 \\
0.40\end{array}$ \\
\hline$S_{3} L P_{S} \pi^{*}{ }_{c c}$ & 4.57 & 0.00 & 4.84 & 0.00 & 4.64 & 0.00 & 4.89 & 0.00 \\
\hline $\mathrm{S}_{4} \pi_{\mathrm{cs}} \pi^{*} \mathrm{cs}$ & 4.81 & 0.01 & 4.86 & 0.01 & 4.23 & 0.01 & 4.90 & 0.01 \\
\hline $\mathrm{S}_{5} \pi_{\mathrm{cc}} \pi^{*} \mathrm{cc}$ & 5.09 & 0.10 & 5.11 & 0.06 & 4.95 & 0.11 & 5.04 & 0.07 \\
\hline $\mathrm{S}_{6} \mathrm{LP}_{\mathrm{o}} \pi^{*} \mathrm{cs}$ & 5.28 & 0.00 & 5.41 & 0.00 & 4.82 & 0.00 & 5.30 & 0.00 \\
\hline $\mathrm{S}_{7} \mathrm{LP}_{\mathrm{s}} \mathrm{Rydb}$ & 4.85 & 0.01 & 5.41 & 0.05 & 5.20 & 0.02 & 5.43 & 0.04 \\
\hline $\mathrm{S}_{8} \pi_{c c} R y d b$ & 5.05 & 0.00 & 5.53 & 0.01 & 5.29 & 0.00 & 5.46 & 0.00 \\
\hline $\mathbf{L P}_{\mathrm{S}}$ & $\pi_{\mathrm{cS}}$ & & & & & $\pi_{c c}^{*}$ & & \\
\hline
\end{tabular}

The experimental MCD (extracted from ref ${ }^{92}$ ) spectrum of 4-TU is depicted in Figure $2 \mathrm{~b}$ (and $3 \mathrm{~b}$ ). This spectrum is characterised by two negative bands (Band I: $326 \mathrm{~nm}$ and Band II: $270 \mathrm{~nm}$ ) well separated in energy $(0.8 \mathrm{eV})$, followed by a small positive band (Band III) at around $230 \mathrm{~nm}$. The computed MCD spectrum of 4-TU (Figure $2 \mathrm{~b}$ ) is in fair agreement with the experimental one. A strong negative peak is predicted at 4.3-4.4 eV (Band I, $S_{2} \pi_{C c} \pi^{*}{ }_{C S}$ ), $0.5 \mathrm{eV}$ blue-shifted with the respect to the experimental band, in analogy with what happens for the absorption spectrum. This feature is followed by a shallow negative feature that we assign to $\pi_{\mathrm{CS}} \pi^{*}{ }_{\mathrm{CS}}$ and $\pi_{c c} \pi^{*}$ cc. The features on the blue-wing are due to electronic transitions involving Rydberg orbitals $\left(\mathrm{S}_{6}\right.$ and $\left.\mathrm{S}_{7}\right)$, whose treatment is not straightforward within a continuum model, and are more sensible to the solvation models and to the size of the basis set.

In the GP, B3LYP stabilizes the dark states compared to CAM-B3LYP: $L P_{S} \pi^{*}{ }_{C C}$ and $L P_{S} R y d b$ are more stable than the bright $\pi_{C S} \pi_{C S}^{*}$ and $\pi_{c c} \pi^{*}{ }_{c c}$ excited states. In solution the effect is less significant, stabilizing mainly the $\mathrm{LP}_{\mathrm{S}} \pi^{*} \mathrm{cc}$. Related with this, in some cases the $\mathrm{LP}_{\mathrm{O}} \pi^{*}$ CS excitation also appears intercalated among the specified states. For what concerns the bright excited states, B3LYP increases the intensity of $\pi_{C C} \pi^{*}{ }_{C C}$, the same result found for 2-TU, improving the agreement with the experimental spectrum (Figure 3e).
Analogously, the second negative feature in the MCD spectrum, is more intense (Figure $3 \mathrm{~b}$ ). than that predicted by CAM-B3LYP, improving the agreement with experiments.

2,4-Thiouracil (2,4-DTU). The presence of two sulphur atoms in 2,4DTU leads to highly-mixed orbitals making a clear assignment of the excited states character much more difficult (see Table 3 ). The number of excited states lying at energies below $6 \mathrm{eV}$ increases up to eight (4 dark and 4 bright states) compared to the monosubstituted uracil (Table 3$)$. The first bright state $\pi_{\mathrm{cc} 2} \pi^{*}{ }_{\mathrm{cs} 4}(4.08 \mathrm{eV})$ and the third $\pi_{\mathrm{CC} 2} \pi^{*}{ }_{\mathrm{CS2} 2}(4.80 \mathrm{eV})$ are similar in energy to the $\mathrm{S}_{1}$ of 4 TU $(4.34 \mathrm{eV})$ and 2-TU $(4.62 \mathrm{eV})$, respectively. Although both excitations share the same bonding orbital $\left(\pi_{\mathrm{CC} 2}\right)$, they differ in the antibonding orbital, which is mainly localized on the $\mathrm{C}=\mathrm{S} 4$ and $\mathrm{C}=\mathrm{S} 2$ for the $\pi_{\mathrm{CC} 2} \pi^{*}{ }_{\mathrm{CS} 4}$ and $\pi_{\mathrm{CC} 2} \pi^{*}{ }_{\mathrm{CS} 2}$ states, respectively. The other two bright states ( 4.44 and $4.92 \mathrm{eV}$ ) are similar, but the excitation involves the other delocalized orbital $\left(\pi_{\mathrm{CC} 1}\right)$. The four excitations present large oscillator strength, 0.12-0.30.

Four dark states are intercalated within these bright states, involving the excitations from the $\mathrm{LP}_{\mathrm{S} 1}$ and $\mathrm{LP}_{\mathrm{S} 2}$ to the $\pi^{*}{ }_{\mathrm{CS} 4}$ and $\pi^{*}{ }_{\mathrm{CS} 2}$ orbitals.

The main solvent effects are similar to those observed for the mono-substituted uracils, namely, the destabilization of the dark states and the change on intensity of the bright states.

The experimental absorption spectrum (Figure $2 \mathrm{f})^{33,92}$ exhibits an intense transition at $360 \mathrm{~nm}(3.44 \mathrm{eV})$, and two close-lying more intense peaks at $\sim 290 \mathrm{~nm}(\sim 4.3 \mathrm{eV})$ and $\sim 260 \mathrm{~nm}(\sim 4.7 \mathrm{eV})$. CAMB3LYP calculations assign the lowest energy peak to $\pi_{\mathrm{CC} 2} \pi^{*}{ }_{\mathrm{CS} 4}(4.04$ $\mathrm{eV})$, whereas the three other transitions $\left(\pi_{\mathrm{CC} 1} \pi^{*}{ }_{\mathrm{CS} 4} / \pi_{\mathrm{CC} 2} \pi^{*}{ }_{\mathrm{CS} 2}\right.$ and $\left.\pi_{\mathrm{CC} 1} \pi^{*}{ }_{\mathrm{CS} 2}\right)$ are responsible of the peaks at 290 and $260 \mathrm{~nm}$. Comparison with the experimental spectra (Figure 2f) shows that the intensity of the $\pi_{\mathrm{CC} 2} \pi^{*}{ }_{\mathrm{CS} 4}$ transition is too large and the relative stability of the higher-lying bright excited states overestimated, leading to a too small energy gap with respect to the $\pi_{\mathrm{CC} 2} \pi^{*}{ }_{\mathrm{CS} 4}$ peak.

In the experimental MCD spectrum, a strong negative feature at $\sim 3.50 \mathrm{eV}$ is followed by a positive one at $\sim 4.40 \mathrm{eV}$. The CAM-B3LYP spectrum is in qualitative agreement with the experimental one. When PCM is included (Figure 2c) the computed spectrum presents two main features: a negative band at $4.0 \mathrm{eV}$ and a positive one at around $4.60-4.80 \mathrm{eV}$, both presenting similar intensity. We thus assign the first band to $\pi_{\mathrm{CC} 2} \pi^{*}{ }_{\mathrm{cs}}$, whereas the other close-lying bright excited states globally provide a positive contribution. It is likely (CPP calculations do not give access to the MCD B terms of the single states) that one of the states would provide a negative signal, as suggested by the shape obtained in absence of PCM, where the three peaks are more separated in energy and a 'sharp' negative peak is present.

B3LYP provides a picture qualitatively similar, on the balance, to that of CAM-B3LYP. However, the four bright transitions responsible for the two lowest energy absorption bands are more separated; $\pi_{\mathrm{CC} 1} \pi^{*}{ }_{\mathrm{CS} 4}$, which at the CAM-B3LYP level is close to $\pi_{\mathrm{CC} 2} \pi^{*}{ }_{\mathrm{CS} 2}$, gets closer to $\pi_{\mathrm{CC} 2} \pi^{*}$ cS4 and shows up in the absorption spectrum. As a consequence the individual contributions of the different transitions to the MCD spectrum are also visible, leading to a more congested spectrum (Figure $3 \mathrm{c}$ ). 
Table 3. 2,4-DTU Vertical Absorption energies ( $\triangle E$, in eV) and oscillator strengths (f) for the different solvation models calculated at the TD-CAMB3LYP/aug-cc-pVDZ and TD-B3LYP/aug-cc-pVDZ level of theory.

\begin{tabular}{|c|c|c|c|c|c|c|c|c|}
\hline 2,4-DTU & \multicolumn{2}{|c|}{ GP } & \multicolumn{2}{|c|}{ PCM } & \multicolumn{2}{|c|}{$4 \cdot \mathrm{H}_{2} \mathrm{O}$} & \multicolumn{2}{|c|}{$\mathrm{PCM}+4 \cdot \mathrm{H}_{2} \mathrm{O}$} \\
\hline CAM-B3LYP & $\Delta \mathrm{E}$ & $f$ & $\Delta \mathrm{E}$ & $f$ & $\Delta \mathrm{E}$ & $f$ & $\Delta \mathrm{E}$ & $f$ \\
\hline $\begin{array}{r}\mathrm{S}_{1} \mathrm{LP}_{\mathrm{s} 2} \pi^{*}{ }_{\mathrm{cs} 4} \\
\mathrm{LP}_{\mathrm{s} 1} \pi^{*}{ }_{\mathrm{cs} 4}\end{array}$ & 3.00 & 0.00 & 3.30 & 0.00 & 3.28 & 0.00 & 3.50 & 0.00 \\
\hline $\mathrm{S}_{2} \pi_{\mathrm{cc} 2} \pi^{*}{ }_{\mathrm{cs} 4}$ & 4.08 & 0.12 & 4.03 & 0.27 & 4.02 & 0.16 & 4.04 & 0.29 \\
\hline $\begin{array}{r}\mathrm{S}_{3} \mathrm{LP}_{\mathrm{s} 2} \pi^{*}{ }_{\mathrm{cs} 2} \\
\mathrm{LP} \mathrm{P}_{\mathrm{s} 1} \pi^{*}{ }_{\mathrm{CS} 2}\end{array}$ & 3.82 & 0.00 & 4.00 & 0.00 & 3.98 & 0.00 & 4.09 & 0.00 \\
\hline $\mathrm{S}_{4} \pi_{\mathrm{CC} 1} \pi^{*}{ }_{\mathrm{CS4}}$ & 4.44 & 0.21 & 4.52 & 0.28 & 4.44 & 0.16 & 4.56 & 0.21 \\
\hline $\mathrm{S}_{5} \pi_{\mathrm{cc} 2} \pi^{*}{ }_{\mathrm{cs} 2}$ & 4.80 & 0.14 & 4.71 & 0.19 & 4.72 & 0.10 & 4.67 & 0.14 \\
\hline $\begin{array}{r}\mathrm{S}_{6} \mathrm{LP}_{\mathrm{s} 2} \pi^{*}{ }_{\mathrm{cs} 4} \\
\mathrm{LP}_{\mathrm{s} 1} \pi^{*}{ }_{\mathrm{cs} 4}\end{array}$ & 4.60 & 0.00 & 4.75 & 0.00 & 4.63 & 0.00 & 4.78 & 0.00 \\
\hline $\mathrm{S}_{7} \pi_{\mathrm{CC} 1} \pi^{*}{ }_{\mathrm{CS} 2}$ & 4.92 & 0.32 & 4.97 & 0.20 & 4.87 & 0.20 & 5.00 & 0.12 \\
\hline $\begin{array}{r}\mathrm{S}_{8} \mathrm{LP}_{\mathrm{s}_{2} \pi^{*}{ }_{\mathrm{cs} 2}} \\
\mathrm{LP}_{\mathrm{s} 1} \pi^{*}{ }_{\mathrm{CS} 2}\end{array}$ & 4.83 & 0.00 & 5.04 & 0.00 & 4.96 & 0.00 & 5.15 & 0.00 \\
\hline B3LYP & $\Delta \mathrm{E}$ & f & $\Delta \mathrm{E}$ & $f$ & $\Delta \mathrm{E}$ & $f$ & $\Delta \mathrm{E}$ & $f$ \\
\hline $\mathrm{S}_{1} \mathrm{LP}_{\mathrm{s} 1} \pi^{*}{ }_{\mathrm{cs} 4}$ & 2.76 & 0.00 & 3.01 & 0.00 & 2.98 & 0.00 & 3.19 & 0.00 \\
\hline $\begin{array}{r}\mathrm{S}_{2} \pi_{\mathrm{CC} 2} \pi^{*}{ }_{\mathrm{CS} 4} \\
\pi_{\mathrm{CC} 1} \pi^{*}{ }_{\mathrm{CS} 4}\end{array}$ & 3.55 & 0.01 & 3.61 & 0.04 & 3.52 & 0.02 & 3.62 & 0.06 \\
\hline $\begin{array}{r}\mathrm{S}_{3} \mathrm{LP}_{\mathrm{s} 2} \pi^{*} \mathrm{cs} 2 \\
\mathrm{LP}_{\mathrm{s} 1} \pi^{*}{ }_{\mathrm{cS} 2}\end{array}$ & 3.47 & 0.00 & 3.65 & 0.00 & 3.54 & 0.00 & 3.70 & 0.00 \\
\hline $\mathrm{S}_{5} \mathrm{LP}_{\mathrm{s} 2} \pi^{*}{ }_{\mathrm{cs} 4}$ & 3.60 & 0.00 & 3.74 & 0.00 & 3.71 & 0.00 & 3.80 & 0.00 \\
\hline $\begin{array}{r}\mathrm{S}_{4} \pi_{\mathrm{CC1}} \boldsymbol{\pi}^{*}{ }_{\mathrm{CS4}} \\
\boldsymbol{\pi}_{\mathrm{CC} 2} \pi^{*}{ }_{\mathrm{CS4}}\end{array}$ & 3.98 & 0.07 & 4.06 & 0.18 & 3.95 & 0.06 & 4.08 & 0.12 \\
\hline $\begin{array}{r}\mathrm{S}_{6} \mathrm{LP}_{\mathrm{s} 2} \pi^{*}{ }_{\mathrm{cs} 2} \\
\mathrm{LP}_{\mathrm{s} 1} \pi^{*}{ }_{\mathrm{cs} 2}\end{array}$ & 3.87 & 0.00 & 4.05 & 0.00 & 3.98 & 0.00 & 4.15 & 0.00 \\
\hline $\begin{array}{r}\mathrm{S}_{7} \pi_{\mathrm{cc} 2} \pi^{*}{ }_{\mathrm{cs} 2} \\
\pi_{\mathrm{CC} 1} \pi^{*}{ }_{\mathrm{CS2} 2}\end{array}$ & 4.51 & 0.22 & 4.41 & 0.24 & * 4.44 & 0.32 & 4.39 & 0.32 \\
\hline $\begin{array}{r}\mathrm{S}_{7} \pi_{\mathrm{CC} 1} \pi^{*}{ }_{\mathrm{cs} 2} \\
\pi_{\mathrm{cC} 2} \pi^{*}{ }_{\mathrm{cs} 2}\end{array}$ & 4.75 & 0.34 & 4.68 & 0.33 & * 4.65 & 0.26 & 4.65 & 0.31 \\
\hline $\mathrm{LP}_{\mathrm{S} 1}$ & & & & & & $\pi$ & & \\
\hline
\end{tabular}

Anoter dark state appears at $4.35 \mathrm{eV}$

\section{Concluding remarks}

In the present study we report a thorough TD-DFT analysis of the OPA and MCD spectra of three different thiouracils, focussing, in particular, on the dependence of our prediction on the environment (gas phase or water) and on the adopted density functional. As a first point, we shall analyse the performance of our methods in reproducing the effect of thionation on the uracil spectra. In all the examined compounds, the lowest energy bright transition is reminiscent of the HOMO $\rightarrow$ LUMO transition of uracil, but for thiouracil $\mathrm{C}=\mathrm{S}$ bonds provide a more substantial contribution than the $\mathrm{C}=\mathrm{O}$ bonds, due to the smaller electronegativity of Sulphur. As previously discussed for thiothymines, ${ }^{44}$ this effect gives account of the red-shift of the absorption spectrum of thiouracils with respect to uracil. CAM-B3LYP predicts that (i) the 4-thionation is more effective than 2-thionation and (ii) the effects (at the 2 and 4 positions) are (approximately) additive. These results, due to the larger conjugation of $\mathrm{C} 4=\mathrm{S}$ bond with $\mathrm{C} 5=\mathrm{C} 6$ double bonds, are fully consistent with the experimental indications. From the quantitative point of view, taking uracil as reference, in the gas phase the lowest bright energy transition is red-shifted in 2-TU by $0.8 \mathrm{eV}$, in 4-TU by $1.1 \mathrm{eV}$ and in 2,4-DTU by $1.5 \mathrm{eV}$. These shifts are close to those derived from the analysis of the experimental absorption maxima $^{33}$ and to those obtained on thiothymine at the CASPT ${ }^{44}$ level (see Table S1). The most significant discrepancy concerns the red-shift of 2-TU, which is overestimated by $\sim 0.3$ $\mathrm{eV}$; interestingly CASPT $2^{44}$ calculations also overestimates the red-shift due to 2-thionation of Thymine. B3LYP predictions are different, since this functional strongly overestimates the red-shift of 2-TU, especially in the gas phase. In GP the lowest energy bright state is red-shifted with respect to that of $4 \mathrm{~T}-\mathrm{U}$, contrary to the experiments in water. Inclusion of solvent effect partially heals this error; at least correctly reproducing the relative position of 2-TU and 4-TU lowest energy peaks (see Table S1).

However, also in water the effect of 2-thionation on the lowest energy bright transition seems overestimated, since B3LYP provides a red-shift of $0.86 \mathrm{eV}$, to be compared with an experimental estimate of $0.5 \mathrm{eV}$. In both 2-TU and 4-TU the HOMO derives from the interaction between the $\pi \pi^{*}$ transitions involving $\mathrm{C} 5=\mathrm{C} 6$ and $\mathrm{C}=\mathrm{S}$ moieties, while the LUMO is more localized on the ring. In 2-TU the two double bonds are not conjugated, the contribution of the $\mathrm{C} 2=\mathrm{S}$ bond to the HOMO is larger and the HOMO $\rightarrow$ LUMO transition acquires a partial formal $(\mathrm{C}=\mathrm{S}) \rightarrow$ ring $\mathrm{CT}$ character. Actually, a simple (Mulliken) test population analysis (Gas Phase TD-B3LYP/6$31 \mathrm{G}(\mathrm{d})$ calculations) indicates that a formal $(\mathrm{C} 2=\mathrm{S}) \rightarrow$ ring charge transfer of ca -0.3 a.u. is associated to the lowest energy bright transition for 2-TU. Both these effects could contribute to explain the poor performance of B3LYP, but only a thorough study of analogous compounds can firmly assess this issue.

On the balance the computed MCD spectra are in good agreement with the experimental ones, confirming the general reliability of our methodological approach. For what concerns CAM-B3LYP, the most significant discrepancy with respect the experiments concerns the relative intensity of the second negative band (Band II) in 4-TU, which is significantly underestimated by our calculations at the CAM-B3LYP level, due to the underestimation of the oscillator strength of the transition(s) responsible for this feature, apparent already in the absorption spectrum. The B3LYP MCD spectrum is in better agreement with experiments for this thiouracil, since this functional better reproduce the relative intensity of the related transitions. These results confirm the importance of a correct estimate of the oscillator strength of the different transitions, an issue often overlooked with respect the analysis of the vertical excitation energies. 
B3LYP, on the other hand, does not reproduce the positive MCD peak of 2-TU, notwithstanding the presence of strong peak in the absorption spectrum.

Comparison with the MCD spectra of uracil shows that a negative peak is always associated to the $\pi_{\mathrm{cc}} \pi^{*}{ }_{\mathrm{CS}}$ transition (the HOMO-LUMO for Uracil). On the contrary, the $\pi_{c c} \pi^{*} c c$ transition is responsible for a positive feature (for uracil it corresponds to the HOMO $\rightarrow$ LUMO+1 transition).

Inclusion of solvent effect does not have a dramatic impact on the computed $M C D$, though, in general, improving the agreement with the position of the experimental peaks. In 2,4-DTU, in particular, in the absence of the solvent the electronic transition would be more separated, producing a spectrum with too many peaks.

For what concerns the interplay with the dark excited states, thionation leads to a significant increase of the energy gap between the lowest energy bright and dark states. As a consequence, at difference with the other pyrimidines, the lowest energy $n \pi^{*}$ transition corresponds always to $S_{1}$, also in water. In this respect, 4-thionation leads to the most stable $\mathrm{n} \pi^{*}$ state (see Table S2), in line with the larger stability of the $\mathrm{n} \pi^{*}$ involving $\mathrm{C} 4=\mathrm{O}$ group in natural pyrimidines. Inclusion of solvent effect is confirmed to lead to a strong relative destabilization of $n \pi^{*}$ transitions, i.e a decrease in the energy gap with the bright state which could a priori accelerate IC and as a consequence intersystem crossing leading to triplet population.

The results here presented confirm that MCD, due its signed nature, is a valuable tool to investigate the relative stability of closely lying excited states, and quadratic response within TDDFT offers a convenient strategy for their calculation. A non phenomenological simulation of $M C D$ shapes require the inclusion of vibrational contributions and in few cases this has been done in literature. ${ }^{96}$ When states become too close, divergences may occur in the quadratic response and stable results can be obtained resorting to the technique of the complex polarization propagator, as it was done here. However, besides these technical problems, in situations of close degeneracy inter-state couplings may affect the shape of electronic spectra and have hardly predictable effects on MCD signals due to the mixing, mediated by vibrations, of contributions with different signs. These effects may be in principle investigated developing a nonadiabatic approach to the computation of MCD spectra. This possibility will be explored in future works.

Furthermore, different tautomers are expected to present different spectral features and comparison between experimental and theoretical MCD could be useful to study their equilibrium both in thiouracils and in other bases.

Figure 2. MCD and OPA spectra for 2-TU $(a, d), 4-T U(b, e)$ and 2,4-DTU $(c, f)$ calculated at the TD-CAM-B3LYP/aug-cc-pVDZ level of theory. Solvation model indicated in the inset. Experimental Spectra extracted from ref ${ }^{92}$ and normalized so to have the intensity of the most intense peak (absolute values) equal to 1.
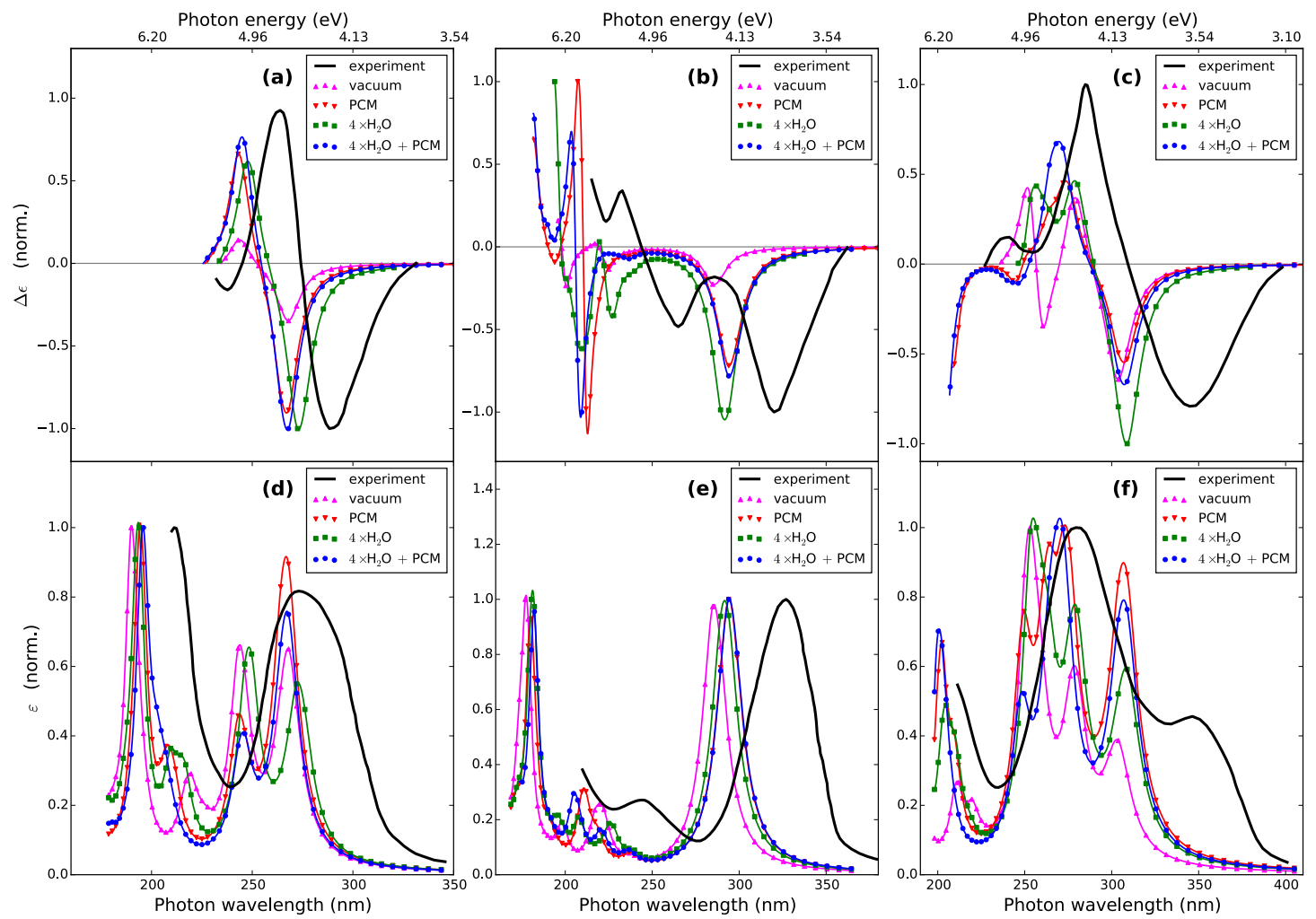
Figure 3. MCD and OPA spectra 2-TU (a,d), 4-TU (b,e) and 2,4-DTU (c,f) calculated at the TD-B3LYP/aug-cc-pVDZ level of theory. Solvation model indicated in the inset. Experimental Spectra extracted from ref ${ }^{92}$ and normalized so to have the intensity of the most intense peak (absolute values) equal to 1.
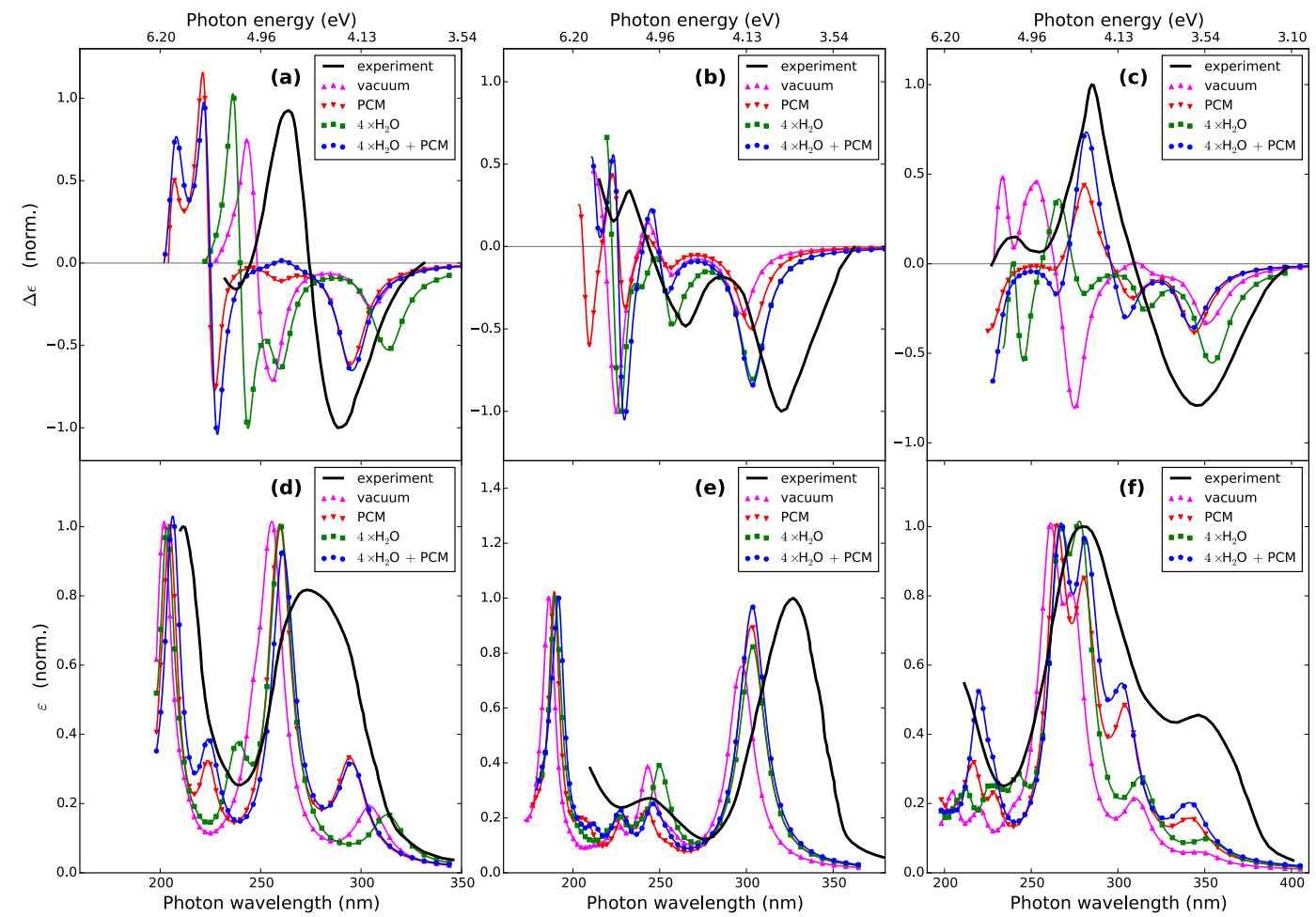


\section{Journal Name}

\section{Acknowledgements}

Financial support from the Swedish Research Council (Grant No. 621-2014-4646) is acknowledged. The calculations were performed on resources provided by the Swedish National Infrastructure for Computing (SNIC) at National Supercomputer Centre (NSC), Sweden. R. I. and F. S. thanks the Progetto Bilaterale CNR/CNRS PICS 2015 for financial support.

\section{Notes and references}

1. M. D. Daniell and J. S. Hill, Aust. NZ J. Surg., 1991, 61, 340348.

2. R. L. Lipson and E. J. Baldes, Arch. Dermatol., 1960, 82, 508516.

3. R. L. Lipson, E. J. Baldes and A. M. Olsen, Natl. Cancer Inst., 1961, 26, 1-11.

4. J. F. Kelly and M. E. Snell, J. Urol., 1976, 115, 150-151.

5. M. R. Hamblin, In: Hamblin MR, Mroz P (eds) Advances in photodynamic therapy: basic, translational and clinical. Artech House, Norwood. 2008.

6. B. Krammer and T. Verwanger, in Applied Photochemistry: When Light Meets Molecules, eds. G. Bergamini and S. Silvi, Springer International Publishing, Cham, 2016, DOI: 10.1007/978-3-319-31671-0_8, pp. 377-396.

7. D. E. J. G. J. Dolmans, D. Fukumura and R. K. Jain, Nat Rev Cancer, 2003, 3, 380-387.

8. R. R. Allison, G. H. Downie, R. Cuenca, X.-H. Hu, C. J. H. Childs and C. H. Sibata, Photodiagnosis Photodyn. Ther., 2004, 1, $27-42$.

9. A. E. O'Connor, W. M. Gallagher and A. T. Byrne, Photochem. Photobiol., 2009, 85, 1053-1074.

10. G. Trigiante and Y. Z. Xu, in Photodynamic Therapy: Fundamentals, Applications and Health Outcomes, ed. A. G. Hugo, Nova Science Publishers, 2015.

11. N. R. Attard and P. Karran, Photochem. Photobiol. Sci., 2012, 11, 62-68.

12. P. Karran and N. Attard, Nat. Rev. Cancer, 2008, 8, 24-36.

13. P. O'Donovan, C. M. Perrett, X. Zhang, B. Montaner, Y.-Z. Xu, C. A. Harwood, J. M. McGregor, S. L. Walker, F. Hanaoka and P. Karran, Science (New York, N.Y.), 2005, 309, 1871-1874.

14. O. Reelfs, P. Karran and A. R. Young, Photochem. Photobiol. Sci., 2012, 11, 148-154.

15. O. Reelfs, P. Macpherson, X. Ren, Y.-Z. Xu, P. Karran and A. R. Young, Nucleic Acids Res., 2011, 39, 9620-9632.

16. S. W. Pridgeon, R. Heer, G. A. Taylor, D. R. Newell, K. O'Toole, M. Robinson, Y. Z. Xu, P. Karran and A. V. Boddy, Br. J. Cancer, 2011, 104, 1869-1876.
17. E. Gemenetzidis, O. Shavorskaya, Y.-Z. Xu and G. Trigiante, J. Dermatol. Treat., 2013, 24, 209-214.

18. A. Massey, Y.-Z. Xu and P. Karran, Current Biology, 2001, 11, $1142-1146$.

19. G. Cui and W.-h. Fang, J. Chem. Phys., 2013, 138, 044315.

20. G. Cui and W. Thiel, J. Phys. Chem. Lett., 2014, 5, 2682-2687.

21. J. P. Gobbo and A. C. Borin, J. Phys. Chem. A, 2013, 117, 5589-5596.

22. J. P. Gobbo and A. C. Borin, Comput. Theor. Chem., 2014, 1040-1041, 195-201.

23. Y. Harada, C. Okabe, T. Kobayashi, T. Suzuki, T. Ichimura, N. Nishi and Y.-Z. Xu, J. Phys. Chem. Lett., 2010, 1, 480-484.

24. Y. Harada, T. Suzuki, T. Ichimura and Y.-Z. Xu, J. Phys. Chem. $B, 2007,111,5518-5524$.

25. J. Jiang, T.-s. Zhang, J.-d. Xue, X. Zheng, G. Cui and W.-h. Fang, J. Chem. Phys., 2015, 143, 175103.

26. S. Mai, P. Marquetand and L. González, J. Phys. Chem. A, 2015, 119, 9524-9533.

27. S. Mai, P. Marquetand and L. González, J. Phys. Chem. Lett., 2016, 7, 1978-1983.

28. S. Mai, M. Pollum, L. Martínez-Fernández, N. Dunn, P. Marquetand, I. Corral, C. E. Crespo-Hernández and L. González, Nat. Commun., 2016, 7, 13077.

29. L. Martinez-Fernandez, I. Corral, G. Granucci and M. Persico, Chem. Sci., 2014, 5, 1336-1347.

30. L. Martinez-Fernandez, L. Gonzalez and I. Corral, Chem. Commun., 2012, 48, 2134-2136.

31. L. Martínez-Fernández, G. Granucci, M. Pollum, C. E. CrespoHernández, M. Persico and I. Corral, Chem. Eur. J., 2017, 23, 2619-2627.

32. M. Pollum and C. E. Crespo-Hernández, J. Chem. Phys., 2014, 140, 071101.

33. M. Pollum, S. Jockusch and C. E. Crespo-Hernandez, Phys. Chem. Chem. Phys., 2015, 17, 27851-27861.

34. M. Pollum, S. Jockusch and C. E. Crespo-Hernández, J. Am. Chem. Soc., 2014, 136, 17930-17933.

35. M. Pollum, L. Martínez-Fernández and C. E. CrespoHernández, in Photoinduced Phenomena in Nucleic Acids I: Nucleobases in the Gas Phase and in Solvents, eds. M. Barbatti, A. C. Borin and S. Ullrich, Springer International Publishing, Cham, 2015, DOI: 10.1007/128_2014_554, pp. 245-327.

36. M. Pollum, L. A. Ortiz-Rodríguez, S. Jockusch and C. E. Crespo-Hernández, Photochem. Photobiol., 2016, 92, 286-292.

37. C. Reichardt and C. E. Crespo-Hernandez, Chem. Commun., 2010, 46, 5963-5965.

38. C. Reichardt and C. E. Crespo-Hernández, J. Phys. Chem. Lett., 2010, 1, 2239-2243.

39. C. Reichardt, C. Guo and C. E. Crespo-Hernández, J. Phys. Chem. B, 2011, 115, 3263-3270. 
40. M. Ruckenbauer, S. Mai, P. Marquetand and L. González, J. Chem. Phys., 2016, 144, 074303.

41. V. Vendrell-Criado, J. A. Saez, V. Lhiaubet-Vallet, M. C. Cuquerella and M. A. Miranda, Photochem. Photobiol. Sci., 2013, 12, 1460-1465.

42. B.-B. Xie, Q. Wang, W.-W. Guo and G. Cui, Phys. Chem. Chem. Phys., 2017, 19, 7689-7698.

43. X. Zou, X. Dai, K. Liu, H. Zhao, D. Song and H. Su, J. Phys. Chem. B, 2014, 118, 5864-5872.

44. S. Bai and M. Barbatti, J. Phys. Chem. A, 2016, 120, 63426350.

45. A. Favre, In Morrison,H. (ed.), Bioorganic Photochemistry: Photochemistry and the Nucleic Acids. Wiley, New York, NY. 1990.

46. M. E. Harris and E. L. Christian, Methods in enzymology, 2009, 468, 127-146.

47. A. Favre and J. L. Fourrey, Acc. Chem. Res., 1995, 28, 375382.

48. S. L. Hiley, V. D. Sood, J. Fan and R. A. Collins, EMBO J., 2002, 21, 4691-4698.

49. A. Favre, C. Saintomé, J.-L. Fourrey, P. Clivio and P. Laugâa, J. Photochem. Photobiol. C, 1998, 42, 109-124.

50. C. Salet, R. V. Bensasson and A. Favre, Photochem. Photobiol., 1983, 38, 521-525.

51. S. J. Milder and D. S. Kliger, J. Am. Chem. Soc., 1985, 107 7365-7373.

52. D. S. Cooper, N. Engl. J. Med., 2005, 352, 905-917.

53. M. S. Masoud, O. H. A. El-Hamid and Z. M. Zaki, Transition Metal Chemistry, 1994, 19, 21-24.

54. M. A. Basinger, J. S. Casas, M. M. Jones, A. D. Weaver and N H. Weinstein, J. Inor. Nuc. Chem., 1981, 43, 1419-1425.

55. B. Ashwood, S. Jockusch and E. C. Crespo-Hernández, Molecules, 2017, 22, 379.

56. M. K. Shukla and J. Leszczynski, J. Phys. Chem. A, 2004, 108, 10367-10375.

57. R. Improta, F. Santoro and L. Blancafort, Chem. Rev., 2016 , 116, 3540-3593.

58. R. Improta and V. Barone, in Photoinduced Phenomena in Nucleic Acids I, eds. M. Barbatti, A. C. Borin and S. Ullrich, Springer International Publishing, 2015, vol. 355, ch. 524, pp. 329-357.

59. F. Buchner, A. Nakayama, S. Yamazaki, H.-H. Ritze and A Lübcke, J. Am. Chem. Soc., 2015, 137, 2931-2938.

60. L. Martinez-Fernandez, A. J. Pepino, J. Segarra-Martí, J. Jovaisaite, I. Vayá, A. Nenov, D. Markovitsi, T. Gustavsson, A. Banyasz, M. Garavelli and R. Improta, J. Am. Chem. Soc., 2017, DOI: 10.1021/jacs.7b01145.

61. F. Santoro, R. Improta, T. Fahleson, J. Kauczor, P. Norman and S. Coriani, J. Phys. Chem. Lett., 2014, 5, 1806-1811.

62. T. Gustavsson, N. Sarkar, I. Vaya, M. C. Jimenez, D. Markovitsi and R. Improta, Photochem. Photobiol. Sci., 2013, 12, 1375-1386.

63. L. Martínez-Fernández, A. J. Pepino, J. Segarra-Martí, A. Banyasz, M. Garavelli and R. Improta, J. Chem. Theor. Comput., 2016, 12, 4430-4439.

64. A. D. Becke, J. Chem. Phys., 1993, 98, 5648.

65. T. H. Dunning, J. Chem. Phys., 1989, 90, 1007-1023.
66. M. J. Frisch, et al Gaussian 09, Revision A.1, in, Gaussian, Inc., Wallingford $C T, 2009$.

67. J. Tomasi, B. Mennucci and R. Cammi, Chem. Rev., 2005, 105, 2999-3094.

68. S. Miertuš, E. Scrocco and J. Tomasi, Chem. Phys., 1981, 55, 117-129.

69. E. Cancès and B. Mennucci, J. Math. Chem., 1998, 23, 309326.

70. E. Cancès, B. Mennucci and J. Tomasi, J. Chem. Phys., 1997, 107, 3032-3041.

71. R. Cammi, L. Frediani, B. Mennucci and K. Ruud, J. CHem. Phys., 2003, 119, 5818-5827.

72. L. Ferrighi, L. Frediani and K. Ruud, J. Phys. Chem. B, 2007, 111, 8965-8973.

73. B. F. Milne and P. Norman, J. Phys. Chem. A, 2015, 119, 53685376.

74. L. Frediani, H. Ågren, L. Ferrighi and K. Ruud, J. Chem. Phys., 2005, 123, 144117.

75. R. Cammi, L. Frediani, B. Mennucci, J. Tomasi, K. Ruud and K. V. Mikkelsen, J. Chem. Phys., 2002, 117, 13-26.

76. Y. He, C. Wu and W. Kong, J. Phys. Chem. A, 2004, 108, 943949.

77. M. Chahinian, H. B. Seba and B. Ancian, Chem. Phys. Lett., 1998, 285, 337-345.

78. T. Gustavsson, Á. Bányász, E. Lazzarotto, D. Markovitsi, G. Scalmani, M. J. Frisch, V. Barone and R. Improta, J. Am. Chem. Soc., 2006, 128, 607-619.

79. F. Santoro, V. Barone, T. Gustavsson and R. Improta, J. Am. Chem. Soc., 2006, 128, 16312-16322.

80. T. Fahleson, J. Kauczor, P. Norman, F. Santoro, R. Improta and S. Coriani, J. Phys. Chem. A, 2015, 119, 5476-5489.

81. R. Improta and V. Barone, J. Am. Chem. Soc., 2004, 126, 14320-14321.

82. T. Yanai, D. P. Tew and N. C. Handy, Chem. Phys. Lett., 2004, 393, 51-57.

83. D. E. Woon and T. H. Dunning, J. Chem. Phys., 1994, 100, 2975-2988.

84. H. Solheim, K. Ruud, S. Coriani and P. Norman, J. Chem. Phys., 2008, 128, 094103.

85. H. Solheim, K. Ruud, S. Coriani and P. Norman, J. Phys. Chem. A, 2008, 112, 9615-9618.

86. M. Krykunov, M. Seth, T. Ziegler and J. Autschbach, J. Chem. Phys., 2007, 127, 244102.

87. DALTON, a molecular electronic structure program, Release Dalton2013 and LSDalton2013. 2013; see http://daltonprogram.org/.

88. F. J. A. Ferrer, R. Improta, F. Santoro and V. Barone, Phys. Chem. Chem. Phys., 2011, 13, 17007-17012.

89. J. Cerezo, F. J. Avila Ferrer, G. Prampolini and F. Santoro, J. Chem. Theor. Comput., 2015, 11, 5810-5825.

90. M. N. Pedersen, E. D. Hedegård, J. M. H. Olsen, J. Kauczor, P. Norman and J. Kongsted, J. Chem. Theor. Comput., 2014, 10, 1164-1171.

91. Z. Rinkevicius, J. A. R. Sandberg, X. Li, M. Linares, P. Norman and H. Ågren, J. Chem. Theor. Comput., 2016, 12, 2661-2667. 
92. N. Igarashi-Yamamoto, A. Tajiri, M. Hatano, S. Shibuya and T. Ueda, Biochimica et Biophysica Acta (BBA) - Nucleic Acids and Protein Synthesis, 1981, 656, 1-15.

93. K. A. Kistler and S. Matsika, J. Phys. Chem. A., 2009, 113, 12396-12403.

94. Q. Li, B. Mennucci, M. A. Robb, L. Blancafort and C. Curutchet, J. Chem. Theor. Comput., 2015, 11, 1674-1682.

95. F. J. Avila Ferrer, F. Santoro and R. Improta, Comput. Theor. Chem., 2014, 1040-1041, 186-194.

96. N. Lin, H. Solheim, X. Zhao, F. Santoro and K. Ruud, J. Chem. Theor. Comput., 2013, 9, 1557-1567. 